\title{
Influence of Bio Fertilizers and Foliar Spray of Spermine and Vermiwash on Growth, Yield and Postharvest Quality of Gerbera (Gerbera jamesonii Hook.) Under Naturally Ventilated Polyhouse
}

\author{
Neelima Palagani ${ }^{1 *}$ and Alka Singh ${ }^{2}$ \\ ${ }^{1}$ Department of Horticulture, College of Horticulture, Dr. YSR Horticultural University, VR \\ Gudem, Andhra Pradesh, India \\ ${ }^{2}$ Dept. of Floriculture and Landscape Architecture, ACHF, NAU, Navsari, Gujarat, India \\ *Corresponding author
}

A B S T R A C T

\begin{abstract}
Keywords
Azotobacter,

Mycorrhiza,

Spermine, Protein,

Peroxidase activity,

Vase life.

Article Info

Accepted:

04 October 2017

Available Online:

10 December 2017 influence of soil inoculation of bio fertilizers (Azotobacter @ 11/ha and VAM @ 2 g/plant) and foliar application of chemicals (spermine and salicylic acid @ 25 and 50 ppm each) and organic growth enhancers (banana pseudo stem sap @ 0.5\% and 1\% and vermiwash @ 4\%) on gerbera. Pooled data of two years indicated that the treatment combination of bio fertilizers along with the foliar spray of spermine @ $25 \mathrm{ppm}$ showed maximum plant growth parameters viz., plant height $(42.87 \mathrm{~cm})$, leaf area index (4.72), suckers per plant (8.42), flower parameters viz., yield per plant $(57.17)$, diameter $(11.41 \mathrm{~cm})$ and fresh weight $(25.11 \mathrm{~g})$ of the flower and physiological parameters like membrane stability index $(75.50 \%)$, protein content $(30.73 \mathrm{mg} / \mathrm{g})$ and peroxidase activity $(48.19 \mathrm{mM} / \mathrm{min} / \mathrm{g}$ protein) in the petal tissue and postharvest life (9.29 days) of gerbera which was at par with or followed by the treatment combination of bio fertilizers along with the foliar spray of vermiwash @ 4\%. Thus, these treatment combinations significantly influenced the growth, yield as well as postharvest quality and life of gerbera.
\end{abstract}

An experiment was conducted during 2013-2015, with factorial concept to study the

\section{Introduction}

Gerbera (Gerbera jamesonii Hook) popularly known as Transvaal daisy, belongs to the family Asteraceae. It is highly popular inthe category of cut flowers and ranks fifth amongst the elite group of top ten cut flowers of the international flower markets (Zheng et al., 2016). Inoculation with bio fertilizers and foliar spray of chemical and organic growth substances can significantly influence the plant growth, yield and quality of cut flowers. Bio fertilizers viz., Azotobacteris free living bacteria, utilize atmospheric nitrogen gas for their cell protein synthesis. This cell protein is then mineralized in soil after the death of Azotobacter cells thereby contributing towards the nitrogen availability of the crop plants (Tchan and New, 1989). Azotobacter has beneficial effects on crop growth and yield through, biosynthesis of biologically active substances and stimulation of rhizospheric microbes (Chen, 2006; Lenart, 2012). Mycorrhiza aid in nutritional management by solubilizing and mobilizing the major and minor plant nutrients and some 
trace elements (Kraepiel et al., 2009) and secretes organic acids, vitamins and growth promoting substances which helps in better plant growth and development (Prasad et al., 2014). Spermine comes under the class of aliphatic polyamines that are ubiquitous in plants and have been associated in wide range of biological processes, including cell division, protein synthesis, DNA replication, differentiation and rhizogenesis (Van den Broek et al., 1994). Vermiwash contains nitrogenous excretory product and growth promoting hormones and essential enzymes for plant growth and also infuses resistance in plants (Kaur et al., 2015). Role of different bio fertilizers, chemicals and natural growth substances as a pre harvest spray in improving the growth, flowering and quality of gerbera has not been explored so far. Hence, this investigation was planned to study the individual and interaction effect of bio fertilizers, chemicals and organic growth substances on growth, yield and postharvest quality of gerbera under naturally ventilated polyhouse.

\section{Materials and Methods}

Present experiment was conducted during 2013-2015, under naturally ventilated polyhouse located at greenhouse complex, Dept. of Floriculture and Landscape Architecture, ACHF, NAU, Navsari. Experiment was laid out in completely randomised design with factorial concept. There were two factors, factor one consisted of two levels as $\mathrm{B}_{0}-$ without bio fertilizers and $\mathrm{B}_{1^{-}}$with bio fertilizers (Azo@11/ha + VAM @ 2g/plant) and factor two consisted of 7 levels of foliar sprays viz., spermine @ 25 ppm $\left(\mathrm{C}_{1}\right)$, spermine @50 ppm $\left(\mathrm{C}_{2}\right)$, salicylic acid @25 ppm $\left(\mathrm{C}_{3}\right)$, salicylic acid @50 ppm $\left(\mathrm{C}_{4}\right)$, banana pseudo stem sap @0.5\% ( $\left.\mathrm{C}_{5}\right)$, banana pseudo stem sap @1.0\% $\left(\mathrm{C}_{6}\right)$ and vermiwash@4\% $\left(\mathrm{C}_{7}\right)$. Thus, there were 14 treatment combinations and were repeated three times. Bio fertilizers viz., Azotobacter was given in the form of drenching and VAM was inoculated in the soil during planting of gerbera. Foliar spray of respective treatments was given one month after transplanting and repeated at six months interval. All the parameters viz., vegetative like plant height (cm), leaf area index, suckers per plant, yield per plant and flowering parameters i.e., diameter of the flower $(\mathrm{cm})$ and fresh weight of the flower $(\mathrm{g})$ and physiological parameters like membrane stability index $(\%)$, protein $(\mathrm{mg} / \mathrm{g})$, peroxidase activity $(\mathrm{mM} / \mathrm{min} / \mathrm{g}$ protein) in the petal tissue and vase life (days) of gerbera were recorded in both the years and data was pooled. Protein and peroxidase activity in the petal tissue were estimated by the methods described by Lowry et al., (1951) and Costa et al., (2002) respectively. The experimental data pertaining to the characters studied were subjected to statistical analysis of variance technique as described by Panse and Sukhatme (1967).

\section{Results and Discussion}

Data depicted in the tabular form revealed that the individual effects of bio fertilizers, foliar spray as well as their interaction showed significant effect on vegetative growth, flower yield and postharvest life of gerbera in both the years as well as pooled data.

\section{Growth and yield}

Growth of the plant in terms of maximum plant height $(44.40,41.33,42.87 \mathrm{~cm})$, leaf area index (4.81, 4.62 and 4.72), number of suckers per plant $(6.23,10.60$ and 8.42$)$ and flower yield per plant $(52.00,62.33$ and 57.17) were recorded in the treatment combination of Azo@ @ 1/ha + VAM @ 2g/plant as soil application and spermine @ $25 \mathrm{ppm}$ as foliar spray $\left(\mathrm{B}_{1} \mathrm{C}_{1}\right)$, which was at par or followed by the treatment combination 
of Azo @ 1 1/ha + VAM @ 2g/plant + vermiwash @ 4\% $\left(\mathrm{B}_{1} \mathrm{C}_{7}\right)$ in first year, second year and pooled data respectively. Azotobacter being a diazotroph, the beneficial response on crops is known due to its atmospheric nitrogen fixing ability and its subsequent mineralization by conversion of elemental form of nitrogen to ammonia as it contains catechol siderophores which have significant percentage of nitrogen and also enhances the uptake of metal ions from the rhizosphere (Yoneyama et al., 2009) (Table 1 and 2).

Further, the ability of Azotobacter to produce B group vitamins (Revillas et al., 2000), indole acetic acid and other plant growth regulators (Lenart, 2012) enhance the root growth and thereby helps in better nutrient absorption (Chen, 2006).

Further, symbiotic relationship of the mycorrhiza with host plants helps in absorption of phosphorus (Miller, 2000), mobilization of organically bound nitrogen (Hodge et al., 2001) and micro and trace elements (Smith and Read, 2008) from soil which are relatively unavailable to the plants and thereby enhance the growth of the plants. Improved growth and flower yield with inoculation of Azotobacter and mycorrhiza were earlier reported in gladiolus (Meenakshi et al., 2015) and in chrysanthemum (Neelima et al., 2015).

Further, spermine is low molecular weight organic polyamine that comes under new class growth regulator (Morgan 1999). Uptake and accumulation of N (Rangan et al., 2014), P (Hewitt, 1963) and K (Hanafy Ahmad et al., 2002) with spermine application has been well documented andalso reported in rose (Farahi et al., 2012). In addition to this, stimulated effect of spermine treatment on plant growth and yield was due to their effect on many metabolic and physiological processes viz., enhanced root growth and floral initiation as per earlier reports (Sood and Nagar, 2003). Vermiwash being mixture of nutrients, vitamins and enzymes (Zambare et al., 2008) have also been reported tofacilitate improved growth and yield in orchid (Karuppaiah and Sendilnathan, 2011) and in rose (Himanshu and Ajit, 2015).

\section{Flower quality}

Gerbera plants inoculated with bio fertilizers (Azo and VAM) and sprayed with spermine @ $25 \mathrm{ppm}\left(\mathrm{B}_{1} \mathrm{C}_{1}\right)$ recorded maximum diameter (12.26 10.54, and $11.41 \mathrm{~cm})$ and fresh weight of flower $(27.04,23.21$ and $25.11 \mathrm{~g}$ ) which was on par with or followed by treatment combination of Azo@11/ha + VAM @2g/plant + vermiwash @ 4\% ( $\left.\mathrm{B}_{1} \mathrm{C}_{7}\right)$ in both the years and pooled data respectively. Improved flower quality parameters can be ascribed to enhanced vegetative growth parameters by inoculation of Azotobacter and VAM and foliar spray of spermine @ 25 ppm and vermiwash @ 4\%. Improved quality parameters have been disclosed earlier with bio fertilizers in chrysanthemum (Neelima et al., 2015) and China aster (Prabhat et al., 2003) and with spermine in rose (Sumathi 2013) (Table 3).

\section{Postharvest physiology and vase life}

Among the interaction of bio fertilizer and foliar spray treatments, significantly maximum protein content $(32.34,29.12$ and $30.73 \mathrm{mg} / \mathrm{g}$ ), peroxidase activity $(48.21,46.38$ and $47.30 \mathrm{mM} / \mathrm{min} / \mathrm{g}$ protein)and membrane stability index $(79.00,71.99$ and $75.50 \%)$ in the petal tissue and vase life $(10.25,8.33$ and 9.29 days) were recorded in the treatment combination of bio fertilizers + spermine @ 25 ppm $\left(\mathrm{B}_{1} \mathrm{C}_{1}\right)$ which was at par with Azo @ 11/ha+VAM@2g/plant + vermiwash@4\% $\left(\mathrm{B}_{1} \mathrm{C}_{7}\right)$ in both the years and pooled data respectively (Tables 4 and 5). 
Table.1 Effect of bio fertilizers, chemicals and organic growth substances on plant height and Leaf Area Index of gerbera var. Alcatraz

\begin{tabular}{|c|c|c|c|c|c|c|c|c|c|c|c|c|c|c|c|c|c|c|}
\hline & \multicolumn{9}{|c|}{ Plant height $(\mathrm{cm})$} & \multicolumn{9}{|c|}{ Leaf Area Index } \\
\hline & \multicolumn{3}{|c|}{ 2013-14 } & \multicolumn{3}{|c|}{$2014-15$} & \multicolumn{3}{|c|}{ Pooled } & \multicolumn{3}{|c|}{ 2013-14 } & \multicolumn{3}{|c|}{$2014-15$} & \multicolumn{3}{|c|}{ Pooled } \\
\hline & $\mathbf{B}_{0}$ & $\mathbf{B}_{1}$ & $\begin{array}{c}\mathrm{C} \\
\text { Mean }\end{array}$ & $\mathbf{B}_{0}$ & $\mathbf{B}_{1}$ & $\begin{array}{c}\text { C } \\
\text { Mean }\end{array}$ & $\mathbf{B}_{0}$ & $\mathbf{B}_{1}$ & $\begin{array}{c}\text { C } \\
\text { Mean }\end{array}$ & $\mathbf{B}_{0}$ & $B_{1}$ & $\begin{array}{c}\text { C } \\
\text { Mean }\end{array}$ & $\mathbf{B}_{0}$ & $\mathbf{B}_{1}$ & $\begin{array}{c}\text { C } \\
\text { Mean }\end{array}$ & $\mathbf{B}_{0}$ & $\mathbf{B}_{1}$ & $\begin{array}{c}\text { C } \\
\text { Mean }\end{array}$ \\
\hline $\mathrm{C}_{1}$ & 40.46 & 44.40 & 42.43 & 39.16 & 41.33 & 40.25 & 39.82 & 42.87 & 41.34 & 3.87 & 4.81 & 4.34 & 3.66 & 4.62 & 4.14 & 3.77 & 4.72 & 4.24 \\
\hline $\mathrm{C}_{2}$ & 39.93 & 40.06 & 39.76 & 39.00 & 39.80 & 39.40 & 39.47 & 39.70 & 39.58 & 3.60 & 4.19 & 3.89 & 3.55 & 4.02 & 3.79 & 3.58 & 4.11 & 3.84 \\
\hline $\mathrm{C}_{3}$ & 39.73 & 39.60 & 39.90 & 36.03 & 39.53 & 37.78 & 37.88 & 39.80 & 38.84 & 3.74 & 4.07 & 3.90 & 3.58 & 4.01 & 3.79 & 3.66 & 4.04 & 3.85 \\
\hline $\mathrm{C}_{4}$ & 39.60 & 40.00 & 39.80 & 38.63 & 39.86 & 39.25 & 39.12 & 39.93 & 39.53 & 3.76 & 3.84 & 3.80 & 3.61 & 3.61 & 3.61 & 3.69 & 3.73 & 3.71 \\
\hline $\mathrm{C}_{5}$ & 40.46 & 41.80 & 41.13 & 34.46 & 39.53 & 37.00 & 37.47 & 40.67 & 39.07 & 3.66 & 4.28 & 3.97 & 3.48 & 4.01 & 3.74 & 3.57 & 4.15 & 3.86 \\
\hline $\mathrm{C}_{6}$ & 39.46 & 41.60 & 40.53 & 38.26 & 40.13 & 39.19 & 38.87 & 40.98 & 39.93 & 3.91 & 4.32 & 4.11 & 3.65 & 4.17 & 3.91 & 3.78 & 4.25 & 4.01 \\
\hline $\mathbf{C}_{7}$ & 39.60 & 43.20 & 41.40 & 39.13 & 40.33 & 39.73 & 38.27 & 41.67 & 39.97 & 3.98 & 4.43 & 4.20 & 3.61 & 4.22 & 3.92 & 3.8 & 4.33 & 4.06 \\
\hline B Mean & 39.89 & 41.52 & - & 37.50 & 40.08 & - & 38.70 & 40.80 & - & 3.79 & 4.28 & - & 3.59 & 4.09 & - & 3.69 & 4.19 & - \\
\hline & B & C & BC & B & $\mathrm{C}$ & BC & B & $\mathrm{C}$ & BC & B & $\mathrm{C}$ & BC & B & $\mathrm{C}$ & BC & B & C & BC \\
\hline C. D.5\% & 0.78 & 1.46 & 2.06 & 0.46 & 0.81 & 1.02 & 0.44 & 0.82 & 1.17 & 0.25 & 0.45 & 0.67 & 0.30 & 0.54 & 0.72 & 0.20 & 0.32 & 0.68 \\
\hline
\end{tabular}

Table.2 Effect of bio fertilizers, chemicals and organic growth substances on suckers/plant and yield/plant of gerbera var. Alcatraz

\begin{tabular}{|c|c|c|c|c|c|c|c|c|c|c|c|c|c|c|c|c|c|c|}
\hline & \multicolumn{9}{|c|}{ Suckers/plant } & \multicolumn{9}{|c|}{ Yield/plant } \\
\hline & \multicolumn{3}{|c|}{ 2013-14 } & \multicolumn{3}{|c|}{ 2014-15 } & \multicolumn{3}{|c|}{ Pooled } & \multicolumn{3}{|c|}{ 2013-14 } & \multicolumn{3}{|c|}{ 2014-15 } & \multicolumn{3}{|c|}{ Pooled } \\
\hline & $\mathbf{B}_{\mathbf{0}}$ & $\mathbf{B}_{1}$ & $\begin{array}{c}\mathrm{C} \\
\text { Mean } \\
\end{array}$ & $\mathbf{B}_{\mathbf{0}}$ & $\mathbf{B}_{1}$ & $\begin{array}{c}\mathrm{C} \\
\text { Mean }\end{array}$ & $\mathbf{B}_{0}$ & $\mathbf{B}_{1}$ & $\begin{array}{c}\mathrm{C} \\
\text { Mean }\end{array}$ & $\mathbf{B}_{0}$ & $\mathbf{B}_{1}$ & $\begin{array}{c}\mathrm{C} \\
\text { Mean } \\
\end{array}$ & $\mathbf{B}_{\mathbf{0}}$ & $\mathbf{B}_{1}$ & $\begin{array}{c}\mathrm{C} \\
\text { Mean } \\
\end{array}$ & $\mathbf{B}_{\mathbf{0}}$ & $\mathbf{B}_{1}$ & $\begin{array}{c}\text { C } \\
\text { Mean }\end{array}$ \\
\hline $\mathbf{C}_{1}$ & 3.36 & 6.23 & 4.80 & 5.36 & 10.60 & 7.98 & 4.37 & 8.42 & 6.39 & 42.00 & 52.00 & 47.00 & 53.00 & 62.33 & 57.67 & 47.50 & 57.17 & 52.33 \\
\hline $\mathbf{C}_{2}$ & 3.00 & 4.73 & 3.86 & 5.60 & 7.66 & 6.63 & 4.30 & 6.20 & 5.25 & 40.67 & 49.33 & 45.00 & 52.33 & 60.33 & 56.33 & 46.50 & 54.83 & 50.67 \\
\hline $\mathrm{C}_{3}$ & 2.70 & 4.86 & 3.78 & 4.20 & 6.60 & 5.40 & 3.45 & 5.73 & 4.59 & 38.33 & 46.67 & 42.50 & 52.00 & 58.67 & 55.33 & 45.17 & 52.67 & 48.92 \\
\hline $\mathrm{C}_{4}$ & 2.56 & 5.43 & 4.00 & 4.20 & 6.03 & 5.11 & 3.38 & 5.73 & 4.56 & 39.67 & 48.67 & 44.17 & 51.67 & 58.33 & 55.00 & 45.67 & 53.50 & 49.58 \\
\hline $\mathrm{C}_{5}$ & 3.50 & 5.20 & 4.35 & 5.91 & 7.43 & 6.66 & 4.70 & 6.32 & 5.51 & 40.00 & 47.67 & 43.83 & 51.00 & 59.00 & 55.00 & 45.50 & 53.33 & 49.42 \\
\hline $\mathrm{C}_{6}$ & 3.66 & 5.81 & 4.73 & 5.43 & 7.70 & 6.56 & 4.55 & 6.75 & 5.65 & 41.00 & 48.33 & 44.67 & 52.00 & 59.67 & 55.83 & 46.50 & 54.00 & 50.25 \\
\hline $\mathrm{C}_{7}$ & 4.06 & 6.06 & 5.06 & 6.30 & 8.10 & 7.20 & 5.18 & 7.08 & 6.13 & 41.33 & 51.33 & 46.33 & 52.33 & 61.00 & 56.67 & 46.83 & 56.17 & 51.50 \\
\hline \multirow[t]{2}{*}{ B Mean } & 3.26 & 5.47 & - & 5.28 & 7.73 & - & 4.28 & 6.60 & - & 40.43 & 49.14 & - & 52.05 & 59.90 & - & 46.24 & 54.52 & - \\
\hline & B & $\mathbf{C}$ & BC & B & $\mathbf{C}$ & BC & B & C & BC & B & C & BC & B & C & BC & B & C & BC \\
\hline C. D.5\% & 0.15 & 0.28 & 0.39 & 0.18 & 0.35 & 0.49 & 0.18 & 0.35 & 0.49 & 0.97 & 1.87 & 2.61 & 1.22 & 2.37 & 3.59 & 0.70 & 1.31 & 1.86 \\
\hline
\end{tabular}


Table.3 Effect of bio fertilizers, chemicals and organic growth substances on diameter and fresh weight of flower of gerbera var. Alcatraz

\begin{tabular}{|c|c|c|c|c|c|c|c|c|c|c|c|c|c|c|c|c|c|c|}
\hline & \multicolumn{9}{|c|}{ Diameter of flower $(\mathrm{cm})$} & \multicolumn{9}{|c|}{ Fresh weight of flower (g) } \\
\hline & \multicolumn{3}{|c|}{ 2013-14 } & \multicolumn{3}{|c|}{$2014-15$} & \multicolumn{3}{|c|}{ Pooled } & \multicolumn{3}{|c|}{ 2013-14 } & \multicolumn{3}{|c|}{$2014-15$} & \multicolumn{3}{|c|}{ Pooled } \\
\hline & $\mathbf{B}_{0}$ & $\mathbf{B}_{1}$ & $\begin{array}{c}\text { C } \\
\text { Mean }\end{array}$ & $\mathbf{B}_{0}$ & $\mathbf{B}_{1}$ & $\begin{array}{c}\text { C } \\
\text { Mean }\end{array}$ & $\mathbf{B}_{0}$ & $\mathbf{B}_{1}$ & $\begin{array}{c}\text { C } \\
\text { Mean }\end{array}$ & $\mathbf{B}_{0}$ & $\mathbf{B}_{1}$ & $\begin{array}{c}\text { C } \\
\text { Mean }\end{array}$ & $\mathbf{B}_{0}$ & $\mathbf{B}_{1}$ & $\begin{array}{c}\text { C } \\
\text { Mean }\end{array}$ & $\mathbf{B}_{0}$ & $\mathbf{B}_{1}$ & $\begin{array}{c}\mathrm{C} \\
\text { Mean }\end{array}$ \\
\hline $\mathrm{C}_{1}$ & 11.28 & 12.26 & 11.77 & 9.07 & 10.54 & 9.80 & 10.18 & 11.41 & 10.79 & 21.51 & 27.04 & 24.27 & 21.69 & 23.21 & 22.45 & 21.60 & 25.11 & 23.36 \\
\hline $\mathrm{C}_{2}$ & 11.20 & 11.82 & 11.51 & 8.82 & 9.52 & 9.17 & 10.01 & 10.67 & 10.34 & 21.06 & 24.34 & 22.70 & 17.82 & 20.97 & 19.40 & 19.44 & 22.65 & 21.05 \\
\hline $\mathrm{C}_{3}$ & 11.07 & 11.63 & 11.35 & 8.85 & 9.62 & 9.23 & 9.97 & 10.63 & 10.30 & 21.27 & 23.89 & 22.58 & 17.35 & 20.07 & 18.71 & 19.31 & 21.98 & 20.65 \\
\hline $\mathrm{C}_{4}$ & 11.01 & 11.71 & 11.36 & 8.75 & 9.74 & 9.24 & 9.89 & 10.73 & 10.31 & 20.41 & 24.36 & 22.38 & 16.93 & 21.87 & 19.40 & 18.67 & 23.11 & 20.89 \\
\hline $\mathrm{C}_{5}$ & 11.23 & 11.59 & 11.41 & 8.65 & 9.76 & 9.21 & 9.95 & 10.68 & 10.31 & 20.65 & 23.95 & 22.30 & 17.84 & 21.21 & 19.52 & 19.25 & 22.58 & 20.91 \\
\hline $\mathrm{C}_{6}$ & 11.19 & 11.43 & 11.31 & 8.49 & 9.99 & 9.24 & 9.84 & 10.71 & 10.28 & 20.58 & 25.35 & 22.96 & 18.12 & 21.96 & 20.04 & 19.35 & 23.66 & 21.50 \\
\hline $\mathbf{C}_{7}$ & 11.02 & 12.20 & 11.61 & 8.21 & 10.32 & 9.26 & 9.62 & 11.27 & 10.44 & 21.44 & 26.49 & 23.96 & 20.28 & 23.20 & 21.74 & 20.86 & 24.85 & 22.85 \\
\hline B Mean & 11.14 & 11.80 & - & 8.69 & 9.93 & - & 9.92 & 10.87 & - & 20.99 & 25.06 & - & 18.43 & 21.78 & - & 19.59 & 23.42 & - \\
\hline & B & C & BC & B & C & $\mathrm{BC}$ & B & C & BC & B & C & BC & B & C & BC & B & C & BC \\
\hline C. D.5\% & 0.037 & 0.067 & 0.098 & 0.030 & 0.056 & 0.079 & 0.019 & 0.035 & 0.50 & 0.46 & 0.86 & 1.22 & 0.36 & 0.67 & 0.95 & 0.28 & 0.53 & 0.75 \\
\hline
\end{tabular}

Table.4 Effect of bio fertilizers, chemicals and organic growth substances on membrane stability index and protein of gerbera var. Alcatraz

\begin{tabular}{|c|c|c|c|c|c|c|c|c|c|c|c|c|c|c|c|c|c|c|}
\hline & \multicolumn{9}{|c|}{ Protein $(\mathrm{mg} / \mathrm{g})$} & \multicolumn{9}{|c|}{ Peroxidase activity (mM/min/g protein) } \\
\hline & \multicolumn{3}{|c|}{ 2013-14 } & \multicolumn{3}{|c|}{$2014-15$} & \multicolumn{3}{|c|}{ Pooled } & \multicolumn{3}{|c|}{ 2013-14 } & \multicolumn{3}{|c|}{$\begin{array}{c}2014-15 \\
\end{array}$} & \multicolumn{3}{|c|}{ Pooled } \\
\hline & $\mathbf{B}_{\mathbf{0}}$ & $\mathbf{B}_{1}$ & $\begin{array}{c}\mathrm{C} \\
\text { Mean } \\
\end{array}$ & $\mathbf{B}_{\mathbf{0}}$ & $\mathbf{B}_{1}$ & $\begin{array}{c}\mathrm{C} \\
\text { Mean } \\
\end{array}$ & $\mathbf{B}_{\mathbf{0}}$ & $\mathbf{B}_{1}$ & $\begin{array}{c}\mathrm{C} \\
\text { Mean } \\
\end{array}$ & $\mathbf{B}_{\mathbf{0}}$ & $\mathbf{B}_{1}$ & $\begin{array}{c}\mathbf{C} \\
\text { Mean } \\
\end{array}$ & $\mathbf{B}_{\mathbf{0}}$ & $\mathbf{B}_{1}$ & $\begin{array}{c}\mathrm{C} \\
\text { Mean } \\
\end{array}$ & $\mathbf{B}_{\mathbf{0}}$ & $\mathbf{B}_{1}$ & $\begin{array}{c}\mathrm{C} \\
\text { Mean } \\
\end{array}$ \\
\hline $\mathbf{C}_{1}$ & 27.57 & 32.34 & 29.96 & 24.32 & 29.12 & 26.72 & 25.95 & 30.73 & 28.34 & 47.29 & 49.13 & 48.21 & 45.52 & 47.24 & 46.38 & 46.40 & 48.19 & 47.30 \\
\hline $\mathbf{C}_{2}$ & 25.67 & 30.26 & 27.96 & 22.34 & 27.03 & 24.69 & 24.00 & 28.65 & 26.32 & 46.77 & 47.29 & 47.03 & 44.50 & 45.62 & 45.06 & 45.63 & 46.45 & 46.04 \\
\hline $\mathrm{C}_{3}$ & 24.37 & 31.46 & 27.92 & 21.12 & 28.23 & 24.68 & 22.75 & 29.85 & 26.30 & 46.25 & 48.31 & 47.28 & 44.46 & 46.13 & 45.29 & 45.35 & 47.22 & 46.29 \\
\hline $\mathrm{C}_{4}$ & 24.54 & 30.90 & 27.72 & 21.59 & 27.87 & 24.73 & 23.07 & 29.39 & 26.23 & 46.67 & 47.91 & 47.29 & 44.85 & 45.71 & 45.28 & 45.76 & 46.81 & 46.28 \\
\hline $\mathrm{C}_{5}$ & 25.06 & 29.64 & 27.35 & 21.68 & 26.41 & 24.05 & 23.37 & 28.03 & 25.70 & 46.67 & 47.06 & 46.87 & 43.81 & 45.41 & 44.61 & 45.24 & 46.24 & 45.74 \\
\hline $\mathrm{C}_{6}$ & 25.37 & 28.97 & 27.17 & 22.14 & 26.35 & 24.25 & 23.76 & 27.66 & 25.71 & 46.74 & 47.63 & 47.19 & 44.86 & 45.15 & 45.01 & 45.80 & 46.39 & 46.10 \\
\hline $\mathrm{C}_{7}$ & 26.36 & 32.25 & 29.31 & 23.24 & 28.32 & 25.78 & 24.80 & 30.28 & 27.54 & 46.87 & 48.54 & 47.71 & 45.32 & 46.31 & 45.82 & 46.10 & 47.43 & 46.76 \\
\hline B Mean & 25.56 & 30.83 & - & 22.35 & 27.62 & - & 23.96 & 29.22 & - & 46.75 & 47.98 & - & 44.76 & 45.94 & - & 45.76 & 46.96 & - \\
\hline & B & $\mathrm{C}$ & $\mathrm{BC}$ & B & $\mathbf{C}$ & $\mathrm{BC}$ & B & $\mathrm{C}$ & $\mathrm{BC}$ & B & $\mathbf{C}$ & $\mathrm{BC}$ & B & $\mathbf{C}$ & $\mathbf{B C}$ & B & $\mathbf{C}$ & $\mathrm{BC}$ \\
\hline C. $D .5 \%$ & 0.67 & 1.26 & 1.78 & 0.76 & 1.42 & 2.00 & 0.64 & 1.20 & 1.69 & 0.31 & 0.58 & 0.83 & 0.25 & 0.45 & 0.66 & 0.17 & 0.32 & 0.46 \\
\hline
\end{tabular}


Table.5 Effect of bio fertilizers, chemicals and organic growth substances on anthocyanin and vase life of gerbera var. Alcatraz

\begin{tabular}{|c|c|c|c|c|c|c|c|c|c|c|c|c|c|c|c|c|c|c|}
\hline & \multicolumn{9}{|c|}{ Membrane Stability Index (\%) } & \multicolumn{9}{|c|}{ Vase life (days) } \\
\hline & \multicolumn{3}{|c|}{ 2013-14 } & \multicolumn{3}{|c|}{ 2014-15 } & \multicolumn{3}{|c|}{ Pooled } & \multicolumn{3}{|c|}{ 2013-14 } & \multicolumn{3}{|c|}{ 2014-15 } & \multicolumn{3}{|c|}{ Pooled } \\
\hline & $\mathbf{B}_{0}$ & $\mathbf{B}_{1}$ & $\begin{array}{c}\text { C } \\
\text { Mean }\end{array}$ & $\mathbf{B}_{\mathbf{0}}$ & $\mathbf{B}_{1}$ & $\begin{array}{c}\text { C } \\
\text { Mean }\end{array}$ & $\mathbf{B}_{0}$ & $\mathbf{B}_{1}$ & $\begin{array}{c}\text { C } \\
\text { Mean }\end{array}$ & $\mathbf{B}_{\mathbf{0}}$ & $\mathbf{B}_{1}$ & $\begin{array}{c}\text { C } \\
\text { Mean }\end{array}$ & $\mathbf{B}_{\mathbf{0}}$ & $\mathbf{B}_{1}$ & $\begin{array}{c}\text { C } \\
\text { Mean }\end{array}$ & $\mathbf{B}_{\mathbf{0}}$ & $\mathbf{B}_{1}$ & $\begin{array}{c}\text { C } \\
\text { Mean }\end{array}$ \\
\hline $\mathbf{C}_{1}$ & 72.01 & 79.00 & 75.51 & 69.32 & 71.99 & 70.66 & 70.67 & 75.50 & 73.08 & 8.30 & 10.25 & 9.27 & 7.08 & 8.33 & 7.70 & 7.69 & 9.29 & 8.49 \\
\hline $\mathbf{C}_{2}$ & 70.14 & 72.34 & 71.24 & 68.01 & 70.00 & 69.01 & 69.07 & 71.17 & 70.12 & 7.63 & 9.15 & 8.39 & 6.34 & 7.96 & 7.15 & 6.99 & 8.56 & 7.77 \\
\hline $\mathbf{C}_{3}$ & 69.59 & 71.39 & 70.49 & 68.41 & 70.31 & 69.36 & 69.00 & 70.85 & 69.93 & 7.73 & 8.55 & 8.14 & 6.26 & 7.65 & 6.96 & 7.00 & 8.10 & 7.55 \\
\hline $\mathrm{C}_{4}$ & 69.11 & 72.10 & 70.61 & 67.87 & 70.34 & 69.11 & 68.49 & 71.22 & 69.86 & 7.93 & 8.64 & 8.28 & 6.17 & 7.68 & 6.93 & 7.05 & 8.17 & 7.61 \\
\hline $\mathrm{C}_{5}$ & 70.12 & 72.87 & 71.50 & 68.73 & 69.99 & 69.36 & 69.43 & 71.43 & 70.43 & 7.53 & 8.68 & 8.10 & 5.57 & 6.64 & 6.10 & 6.55 & 7.66 & 7.11 \\
\hline $\mathrm{C}_{6}$ & 69.77 & 73.55 & 71.66 & 67.81 & 71.21 & 69.51 & 68.79 & 72.38 & 70.59 & 7.63 & 8.84 & 8.23 & 5.92 & 7.01 & 6.46 & 6.78 & 7.93 & 7.35 \\
\hline $\mathbf{C}_{7}$ & 73.81 & 73.72 & 73.76 & 69.28 & 71.41 & 70.35 & 71.54 & 72.57 & 72.05 & 8.26 & 9.58 & 8.95 & 6.44 & 7.96 & 7.20 & 7.36 & 8.78 & 8.07 \\
\hline B Mean & 70.65 & 73.57 & - & 68.49 & 70.75 & - & 69.57 & 72.16 & - & 7.86 & 9.10 & - & 6.25 & 7.60 & - & 7.06 & 8.35 & - \\
\hline & B & C & BC & B & C & BC & B & C & BC & B & C & BC & B & C & BC & B & C & BC \\
\hline C. D.5\% & 0.89 & 1.66 & 2.53 & 0.31 & 0.58 & 0.82 & 0.44 & 0.82 & 1.77 & 0.10 & 0.20 & 0.28 & 0.05 & 0.10 & 0.14 & 0.06 & 0.12 & 0.14 \\
\hline
\end{tabular}


Improved postharvest parameters might be due to enhanced overall food and nutrient status of the flowers and greater development of conducting tissues which facilitated higher flower fresh weight in inoculated plants. Further, application of spermine have been known to prevent senescence through inhibition of protein degradation (Baraniak and Kostecka, 1999), by maintaining the membrane stability (Apelbaum et al., 1981) through binding with membrane phospholipids and other anion component of the membranes (Katarzyna et al., 2012). Thus, retained flower fresh weight, higher protein content, peroxidase activity and membrane stability in the petal tissue with Azotobacter and VAM inoculation and foliar spray of spermine@25 ppm and vermiwash @ 4\% treatments ultimately resulted in to enhanced vase life of gerbera. Similar results of enhanced vase life were earlier reported with bio fertilizes in gerbera (Barreto and Jagtap, 2002) and with spermine in gladiolus (Nahed et al., 2009) and rose (Sumathi, 2013) and with vermiwash in gladiolus (Samir, 2016).

\section{Acknowledgement}

The first author is the recipient of Inspire fellowship from the Department of Science and Technology (DST), New Delhi for PhD studies. Hence, DST, New Delhi is highly acknowledged as this research article is a part of her Ph.D research work.

\section{References}

Apelbaum A, Bugoon A C, Anderson J D, Lieberman M, Benarie R and Mattoo A K. 1981. Polyamines inhibit biosynthesis of ethylene in higher plant tissue and fruit protoplast. Plant Physiology, 68: 453-56.

Baraniak B and Kostecka M 1999. Inhibition of protease activity in a protein supplement made from alfalfa and sunflower leaves by polyamines and growth regulators. Annales, 54: 187-94.

Barreto M S and JagtapK B. 2002. Studies on polyhouse gerbera substrate. "Floriculture research trend in India." Proceedings of the national symposium on Indian floriculture in the new millennium, LalBagh, Bangalore, 2527, February, 2002, 173-76.

Chen J. 2006. The combined use of chemical and organic fertilizers and/or bio fertilizer for crop growth and soil fertility. International workshop on Sustained Management of the SoilRhizosphere System for Efficient Crop Production and Fertilizer Use, Thailand, pp. 1-10.

Costa H, Gallego S M and Tomaro M L. 2002. Effect of UV-B radiation on antioxidant defense system in sunflower cotyledons. Plant Science, 62: 939- 45.

Farahi HF, Khalingi A, Kholdbarin B, Akbarboojar MM, Eshghi S, Kavoosi B and Aboutalebi A. 2012. Influence of exogenous spermidine on quality properties and vase life of rose (Rosa hybrida cv. Dolcvita). Annals of Biological Research, 3(10): 4758-63.

Hanafy Ahmed A H, Gad M A, Hassan M A and Amin 2002. Improving growth and chemical composition of Myrtus communis grown under soil salinity conditions by polyamines foliar application. Proc. Minia $1^{\text {st }}$ conf. for Agric and Environ. Sci., Minia, Egypt, March, 25-28.

Hewitt E J. 1963. The essential nutrients elements: Requirements and interactions in plants. In: Steward, F.C. (eds). Plant Physiol., Atreatise, Academic, NY, pp. 137-60.

Himanshu Trivedi and Ajit Kumar 2015. Response of bio-enhancers on growth and flowering in rose (Rosa hybrida)cv. Grand Gala. International Journal of 
Basic and Applied Agricultural Research, 13(1): 31-6.

Hodge A, Campbell C D and Fitter A H. 2001. An arbuscular mycorrhizal fungus accelerates decomposition and acquires nitrogen directly from organic material. Nature, 413: 297-99.

Karuppaiah P and Sendilnathan R. 2011. Studies on the effect of foliar application of organic nutrients on the growth, flowering and flower quality of Dendrobium orchid cv. Sakura Pink. Advances in Plant Science, 24(2): 60912.

Katarzyna Rubinowska, Elibieta Pogroszewska and Waadysaaw Michaaek. 2012. The effect of polyamines on physiological parameters of post-harvest quality of cut stems of rose 'Red Berlin'. Acta Sci. Pol., 11(6): 81-93.

Kaur P, Bhardwaj M and Babbar I. 2015. Effect of vermicompost and vermiwash on growth of vegetables. Research Journal of Animal Veterinary and Fishery Science, 3(4): 9-12.

Kraepiel A M L, Chiffoleau J F, Martin J M and Morel F M M. 2009. Geochemistry of trace metals in the Gironde estuary. GeochimCosmochimActa, 61: 1421-36.

Lenart A. 2012. Occurrence, characteristics and genetic diversity of Azotobacter chroococcum in various soils of southern Poland. Poland Journal of Environmental Study, 21(2): 415-24.

Lowry O H, Rosbrough N J, Farr A L and Randall R J. 1951. Protein measurement with the folin phenol reagent. Journal of Biology and Chemistry, 193: 265-75.

Meenakshi Basoli, Prabhat Kumar and Santosh Kumar. 2015. Impact of integrated nutrient management on growth and flowering of gladiolus (Gladiolus hybrida) cv. Novalux. Indian Journal of Agricultural Science, 85(5): 35-9.
Miller M H. 2000. Arbuscular mycorrhizae and the phosphorus nutrition of maize: A review of Guelph studies. Canadian Journal of Plant Pathological Science, 80:47-52.

Morgan D M. 1999. Polyamines: an overview. Molecular Biotechnology, 11: 229-50.

Nahed A A, TahaLobna G and Ibrahim Soad M M. 2009. Some studies on the effect of putrescine, ascorbic acid and thiamine on growth, flowering and some chemical constituents of gladiolus plants at Nubaria. Ozean Journal of Applied Science, 2(2): 1943-2429.

Neelima P, Barad A V, Manisha Shinde and Nilima Bhosale 2015. Response of bio fertilisers and organic manures on growth, flowering, yield and quality of chrysanthemum. Green Farming International Journal, 6(3): 565-68.

Panse V G and Sukhatme P V. 1967. Statistical Methods for Agricultural Workers. ICAR Pub., New Delhi.

Prabhat Kumar, Raghava S P S and Mishra R L. 2003.Effect of bio fertilizers on growth and yield of China aster. Journal of Ornamental Horticulture, 6(2): 85- 8.

Prasad S J, Subhash R R, Narayan R R and Uma, R A. 2014. Isolation, screening and characterization of Azotobacter from rhizospheric soils for different plant growth promotion (PGP) and antagonistic activities and compatibility with agrochemicals: An in vitro study. Ecology, Environment and Conservation, 20(3): 959-66.

Rangan P, Subramani R, Rajesh Kumar, Singh A K and Singh R. 2014. Recent advances in polyamine metabolism and abiotic stress tolerance. International Biological and Medicinal Research, 9: 1-9.

Revillas J J, Rodelas B, PozoC, Martinez Toledo M V and Gonzalez Lopez J. 
2000.Production of B- group vitamins by two Azotobacter strains with phenolic compounds as sole carbon source under diazotrophic and adiazotrophic conditions. Journal of Applied Microbiology, 89(3): 486-93.

Samir K T. 2016. Effect of plant growth regulators, vermiwash and cow urine on vegetative growth, flowering, corm production and vase life of gladiolus var. Candyman. Ph. D. thesis submitted to IGKV, Raipur, Chhattisgarh.

Singh A, Kumar J and Kumar P. 2008. Effects of plant growth regulators and sucrose on postharvest physiology, membrane stability and vase life of cut spikes of gladiolus. Plant Growth Regulation, 55: 221-29.

Smith S E and Read D J. 2008. Mycorrhizal symbiosis. $3^{\text {rd }}$ edition. Academic press, London, U K., 800 Pp.

Sood S H and Nagar P K. 2003. The effect of polyamines on leaf senescence in two diverse rose species. Plant Growth Regulation, 39: 155-60.

Sumathi T. 2013. Effect of different chemicals and natural preservatives as a pre and postharvest spray on greenhouse rose cv. Samurai. M. Sc. thesis submitted to Navsari Agricultural University, Gujarat.

Tchan Y T and New PB. 1989.
Azotobacteraceae. In: Holt JG, Williams \& Wilkins (Eds.) Bergey's Manual of Systematic Bacteriology Volume 1. Baltimore, USA, pp. 220-29.

Van den Broeck D, Van Den S D, Van Montague M and Caplan A. 1994. A group of chromosomal proteins in specifically released by spermine and loses DNA-binding activity upon phosphorylation. Plant Physiology, 106: 559-66.

Yoneyama F, Yamamoto M, Hashimoto W and Murata K. 2009. Azotobacter vinelandii gene clusters for two types of peptidic and catechol siderophores produced in response to molybdenum. Journal of Applied Microbiology, 111: 932-38.

Zambare V P, Padul M V, Yadav A A and Shete T B. 2008. Vermiwash: Biochemical and microbiological approach as ecofriendly soil conditioner. Journal of Agriculture and Biological Science, 3: 1-5.

Zheng Chen Yanhui, Na Lu, Min Wu, Chaoqun $\mathrm{Wu}$ and Shaoyun He. (2016). Genetic diversity and relationships of 48 cultivars of Gerbera jamesonii Adlam in China revealed by microsatellite markers. Bangladesh $J$. Bot., 45(3): 641-648.

\section{How to cite this article:}

Neelima Palagani and Alka Singh. 2017. Influence of Bio Fertilizers and Foliar Spray of Spermine and Vermiwash on Growth, Yield and Postharvest Quality of Gerbera (Gerbera jamesonii Hook.) Under Naturally Ventilated Polyhouse. Int.J.Curr.Microbiol.App.Sci. 6(12): 245-253. doi: https://doi.org/10.20546/ijcmas.2017.612.030 